\title{
Successful Modified Nikaidoh Procedure (Pivot Rotation) in a Patient with Double Outlet Right Ventricle and Pulmonary Atresia: Case Report
}

\author{
Jae Jun Lee, M.D., Ok Jeong Lee, M.D., Ji-Hyuk Yang, M.D., Ph.D., Tae-Gook Jun, M.D., Ph.D. \\ Department of Thoracic and Cardiovascular Surgery, Samsung Medical Center, Sungkyunkwan University School of Medicine, Seoul, Korea
}

\section{ARTICLE INFO}

Received July 29, 2020

Revised October 26, 2020

Accepted October 28, 2020

Corresponding author

Tae-Gook Jun

Tel 82-2-3410-3483

Fax 82-2-3410-6986

E-mail tg.jun@samsung.com

ORCID

https://orcid.org/0000-0002-5161-4665
Double outlet right ventricle (DORV) with pulmonary atresia (PA) is an uncommon congenital disease. Because of anatomical and physiological anomalies in the systemic and pulmonary circulation, corrective surgery may be challenging. We present the case of a patient with DORV and PA. This condition was successfully corrected using a modified Nikaidoh procedure, resulting in reduced obstruction of the left ventricular outflow tract.

Keywords: Congenital heart disease, Pulmonary atresia, Double outlet right ventricle, Arterial switch operation, Modified Nikaidoh operation, Case report

\section{Case report}

\section{Case}

A patient was born at a gestational age of 41 weeks with a body weight of $3.8 \mathrm{~kg}$. The patient was diagnosed with double outlet right ventricle (DORV) and pulmonary atresia (PA). After birth, the patient's hemodynamic status was stable, without cyanosis. Echocardiography confirmed DORV, a moderate-to-large ventricular septal defect (VSD, approximately $6 \mathrm{~mm}$ ), a secundum atrial septal defect (approximately 9-10 $\mathrm{mm}$ ), PA with an intact main pulmonary trunk, left-to-right dominant pulmonary ductus arteriosus shunt flow, and coronary anatomy classifications of 1LCX2R and Yacoub type A (Fig. 1).

A 1-stage total corrective procedure with a long cardiopulmonary bypass (CPB) time was expected to be an excessive burden on the patient. Therefore, we chose to perform a 2-stage correction. At 12 days of age, the patient had a body weight of $4 \mathrm{~kg}$, and he first underwent patent ductus arteriosus ligation and a left-modified BlalockTaussig shunt procedure (left subclavian to left pulmonary artery), performed using a Gore-Tex 4-mm graft through a left posterolateral thoracotomy incision in the fourth inter-

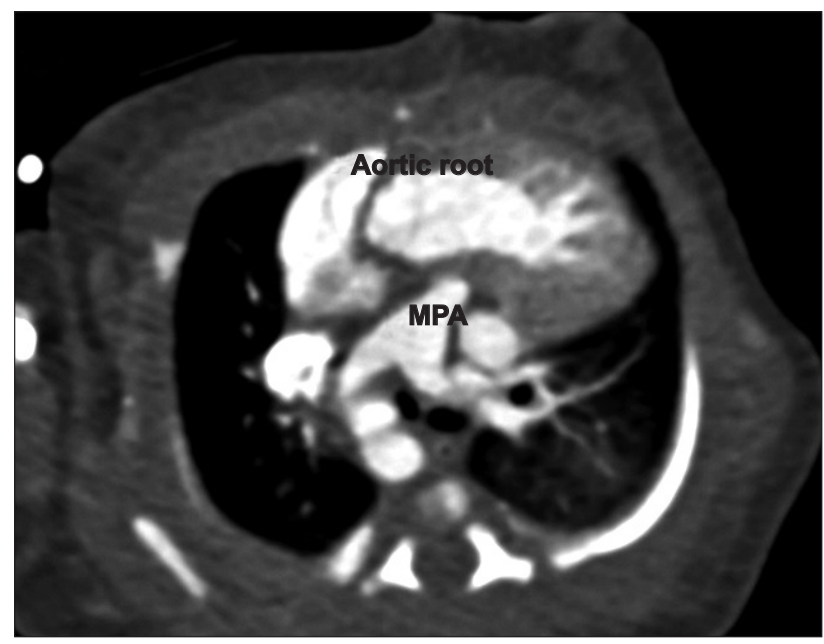

Fig. 1. Preoperative heart computed tomography findings depicting the great artery (aortopulmonary) relationship. MPA, main pulmonary artery.

costal space.

The patient was discharged on postoperative day 60 with left vocal cord palsy and hoarseness, the assumed cause of which was left recurrent laryngeal nerve injury. After discharge, we were unable to follow up with the patient, who 
did not visit the hospital. At 11 months, the patient's body weight was $8 \mathrm{~kg}$, and he returned to the hospital for a total corrective operation.

In the second operation, a modified Nikaidoh procedure was successfully performed. The CPB, aortic cross-clamp, and total operation times were 200 minutes, 161 minutes, and 350 minutes, respectively.

The patient was extubated on postoperative day 7 but was intubated 6 hours later because of sudden hemoptysis. The cause of the sudden hemoptysis was unknown, but was presumed to be bleeding in the bronchial artery branches or airway injury related to the endotracheal tube.

After this intubation, the patient was hemodynamically stable with no bleeding, and he was successfully extubated 4 days later. The patient's condition was stable, with an oxygen saturation level of $99 \%$ without respiratory support, a blood pressure of $100 / 60 \mathrm{~mm} \mathrm{Hg}$, and a heart rate of 130 beats/min. No cardiac defect was observed at discharge. We followed up with the patient until he was 18 months old, during which time he was healthy without any cardiac problems.

\section{Operative procedure}

The operation was performed through a standard median sternotomy. After thymectomy, the existing left-modified Blalock-Taussig shunt was ligated before CPB was initiated. CPB was established with bicaval cannulation, and the patient was cooled to $23^{\circ} \mathrm{C}$ (deep hypothermia). The pulmonary arteries were extensively mobilized to facilitate a Lecompte maneuver. After cardiac arrest with cold blood cardioplegia, the right atrium was opened with an oblique incision, and the VSD was inspected. Both great arteries were mobilized; the aorta was transected above the sinotubular junction, and the pulmonary artery was transected just above the annulus. The right coronary artery button was cut, and the proximal part of the right coronary artery was dissected. After inspection of the right ventricular outflow tract (RVOT), a transverse incision was made below the level of the aortic valve and was extended circumferentially around the aortic valve except immediately below the left coronary artery, maintaining a muscle rim of 3-5 $\mathrm{mm}$. As such, we left the left coronary artery intact. After identification of the left outflow tract immediately below the pulmonary annulus, we transected the septum and pulmonary valve annulus to extend the VSD and create space for the aortic root. The aortic root was rotated clockwise and was anastomosed to the rim of the pulmonary annulus and the transected edges of the infundibular septum (pivot rotation). The new plane of the VSD (from the crest of the original VSD to the free edge of the aortic root circumference) was closed with bovine pericardium using running sutures. After the Lecompte maneuver, the distal aorta was anastomosed to the proximal aorta with a running suture, and a cardioplegic solution was infused into the inflated aortic root. The exact desired site of the right coronary artery was marked, and the right coronary button was anastomosed to the aortic root. The RVOT was reconstructed with a pulmonary homograft conduit (Fig. 2).

This study was approved by the Institutional Review Board of Sungkyunkwan University (IRB approval no., 2020-10-180). The patient provided verbal informed consent for the publication of his clinical details and images.
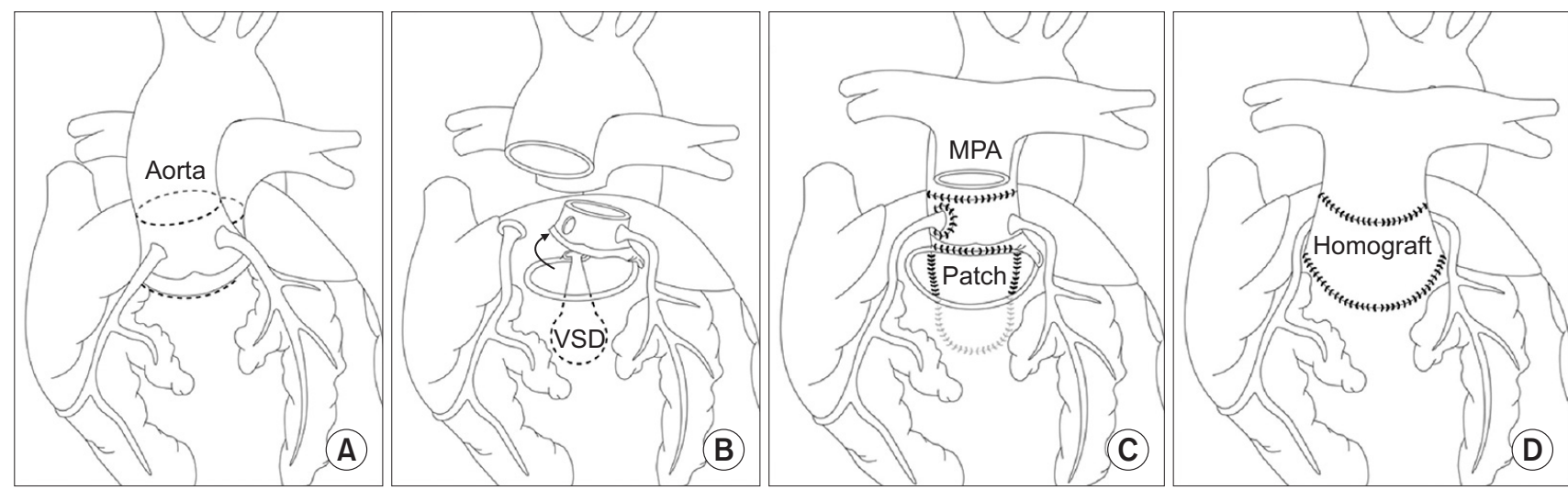

Fig. 2. Operative procedure. (A) Transverse incision of the aorta below the aortic valve and aortic root harvest. (B) Aortic root pivot rotation and the Lecompte maneuver. (C) Left ventricular outflow tract reconstruction with VSD extension and patch closure. (D) MPA reconstruction with a pulmonary homograft. VSD, ventricular septal defect; MPA, main pulmonary trunk. 


\section{Discussion}

DORV is generally defined as a condition in which over $50 \%$ of the aortic root and the pulmonary artery arise from the morphologic right ventricle (RV). Depending on the extent of aorta override, left ventricular outflow tract (LVOT) reconstruction may be needed for patients in addition to creation of a baffle, as in our case (Fig. 3A). Various surgical methods and modifications exist for biventricular repair and outflow tract reconstruction.

In 1968, Rastelli et al. [1] reported the successful anatomical reconstruction of the RVOT in a patient with transposition of the great arteries (TGA), VSD, and subvalvular pulmonary stenosis. That procedure involved the reconstruction and bypass of the biventricular outflow tract using a homograft and Teflon tunnel. Two complications that may arise after the Rastelli procedure are dynamic LVOT obstruction due to the presence of a long intracardiac tunnel and the frequent need for late reoperation due to conduit failure [2].

In 1980, Lecompte et al. [3] performed an anatomical correction of TGA with VSD and pulmonary stenosis through the réparation a l'étage ventriculaire (REV) procedure. This technique is a method of anatomic correction in which the left ventricle is connected to the aorta and the $\mathrm{RV}$ to the pulmonary artery via the rearrangement of the pulmonary trunk behind the ascending aorta to the anterior side of the aorta. Compared to the Rastelli method, the REV procedure results in a straighter and shorter intracardiac LVOT via resection of the conal septum. Through direct reimplantation of the pulmonary trunk into the RV, the REV approach also avoids the need for frequent reoperation due to conduit failure.

In 1984, Nikaidoh [4] first utilized a new corrective method for TGA with VSD and pulmonary stenosis that was based on aortic root translocation. Posterior movement of the aortic root, with or without rotation, is essential for the Nikaidoh procedure. Complete repositioning of the aortic root offers fine, delicate alignment of the LVOT, which can reduce aortic valve insufficiency. In a 15 -year midterm follow-up study of the Nikaidoh operation, the rate of freedom from reoperation due to LVOT-related problems was $100 \%$, while the same rate due to RVOT-related problems was $64 \%$. However, with regard to the incidence of RVOT obstruction, the Nikaidoh operation was reported to have no significant advantage over other operations, including the Rastelli and REV procedures [5]. The movement of the aortic root may trigger kinking of the coronary artery. Careful mobilization of the aortic root and coronary arteries is needed to prevent any interruption or kinking of the coronary arteries.

Certain modifications of the Nikaidoh procedure, which involve the Lecompte maneuver and coronary artery reimplantation, could relieve tension and malalignment of the coronary artery. Kramer et al. [6] reported results of a modified Nikaidoh procedure in cases of TGA with VSD; the 5-year rate of freedom from reoperation due to LVOT-related problems was $100 \%$, while the rates of freedom from reoperation and reintervention (catheter dilatation) due to RVOT-related problems were $87 \%$ and $81 \%$, respectively.

The patient in the present case had DORV with PA and a long distance between the aorta and the interventricular septum, interposed by the pulmonary root (Fig. 3A). To reduce postoperative coronary artery- and LVOT-related complications, we performed a modified Nikaidoh procedure involving the Lecompte maneuver, coronary artery transfer, and aortic root translocation; this procedure al-
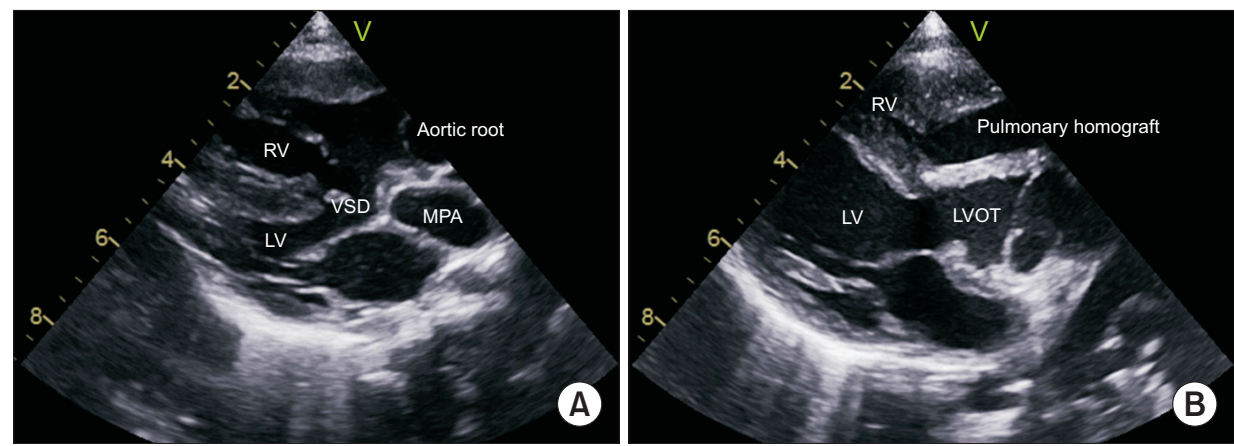

Fig. 3. Preoperative and postoperative echocardiography. (A) Preoperative echocardiography. The aorta interposed by the pulmonary root was distant from the interventricular septum. (B) Postoperative echocardiography. Aortic pivot rotation provided good alignment of the left ventricular outflow tract. LV, left ventricle; RV, right ventricle; VSD, ventricular septal defect; MPA, main pulmonary artery; LVOT, left ventricular outflow tract. 
lowed for a shortened LVOT, orthotopic positioning of the RVOT, and natural intracardiac geometry [7]. Because coronary artery detachment and transfer can introduce coronary stenosis at the anastomotic site as well as coronary artery kinking, we modified the Nikaidoh procedure by partially resecting the aortic root to allow for clockwise pivot rotation of the root and did not detach the left coronary artery, which was sufficiently long. However, the benefits of the Nikaidoh procedure in cases of pulmonary hypoplasia or PA are not certain. In this patient, despite the diagnosis of PA, the main pulmonary root was not very small (diameter, $10.9 \mathrm{~mm}$; Z-score, -1.12). The size of the pulmonary root was almost half that of the aortic root. We believe that the modified Nikaidoh procedure can be beneficial in creating sufficient space for LVOT placement and enabling better alignment of the new LVOT [8]. Other options for root translocation exist, such as the half-turned truncal switch procedure (the Yamagishi procedure). This procedure may be an option for cases of TGA with pulmonary stenosis or PA. However, this procedure involves complicated and precise techniques (en bloc resection and anastomosis of the infundibulum, as well as the implantation of 2 coronary arteries) and requires specific surgical experience. The patient in the present case had unrestricted VSD and PA. Thus, the benefits of the half-turned truncal switch procedure were uncertain. Postoperative echocardiography revealed a geometrically well-aligned LVOT, and the postoperative clinical course was satisfactory (Fig. 3B).

For the abovementioned procedures, RVOT-related complications are the major causes of reoperation. The RVOT reconstruction method is an important factor in lowering complications and mortality after surgery. Generally, the RVOT is reconstructed using a valve-type conduit, which has unsatisfactory long-term durability due to calcification, degeneration, and size mismatch. In a study by Raju et al. [9], RVOT reconstruction with a transannular patch helped lower the reoperation rate in the intermediate follow-up period. However, in the long term, right ventricular failure will occur due to pulmonary regurgitation. Similarly, we used a homograft for the RV-pulmonary artery connection, and we cannot avoid future reoperation [10].

In conclusion, in patients with DORV with PA, as in this case of overriding LVOT, modification of the Nikaidoh procedure with aortic root pivot rotation offers some benefits. These benefits stem from the anatomically suitable position of the ventricular outflow tract (particularly the LVOT) attained via minimal manipulation of aortic root structures, leading to lower complications and better outcomes.

\section{Conflict of interest}

No potential conflict of interest relevant to this article was reported.

\section{ORCID}

Jae Jun Lee: https://orcid.org/0000-0003-0466-7630

Ok Jeong Lee: https://orcid.org/0000-0001-9234-1424

Ji-Hyuk Yang: https://orcid.org/0000-0002-7349-6778

Tae-Gook Jun: https://orcid.org/0000-0002-5161-4665

\section{References}

1. Rastelli GC, Wallace RB, Ongley PA. Complete repair of transposition of the great arteries with pulmonary stenosis: a review and report of a case corrected by using a new surgical technique. Circulation 1969;39:83-95.

2. Dearani JA, Danielson GK, Puga FJ, Mair DD, Schleck CD. Late results of the Rastelli operation for transposition of the great arteries. Semin Thorac Cardiovasc Surg Pediatr Card Surg Annu 2001;4:3-15.

3. Lecompte Y, Neveux JY, Leca F, et al. Reconstruction of the pulmonary outflow tract without prosthetic conduit. J Thorac Cardiovasc Surg 1982;84:727-33.

4. Nikaidoh H. Aortic translocation and biventricular outflow tract reconstruction: a new surgical repair for transposition of the great arteries associated with ventricular septal defect and pulmonary stenosis. J Thorac Cardiovasc Surg 1984;88:365-72.

5. Marcelletti C, Mair DD, McGoon DC, Wallace RB, Danielson GK. The Rastelli operation for transposition of the great arteries: early and late results. J Thorac Cardiovasc Surg 1976;72:427-34.

6. Kramer P, Ovroutski S, Hetzer R, Hubler M, Berger F. Modified Nikaidoh procedure for the correction of complex forms of transposition of the great arteries with ventricular septal defect and left ventricular outflow tract obstruction: mid-term results. Eur J Cardiothorac Surg 2014;45:928-34.

7. Jeon JH, Seong YW, Kim WH, Chang HW, Chung ES, Kwak JG. Modified Nikaidoh procedure for patient with TGA, restrictive VSD, and PS. Korean J Thorac Cardiovasc Surg 2009;42:87-91.

8. Lee HP, Bang JH, Baek JS, Goo HW, Park JJ, Kim YH. Aortic root translocation with arterial switch for transposition of the great arteries or double outlet right ventricle with ventricular septal defect and pulmonary stenosis. Korean J Thorac Cardiovasc Surg 2016;49:190-4.

9. Raju V, Myers PO, Quinonez LG, et al. Aortic root translocation (Nikaidoh procedure): intermediate follow-up and impact of conduit type. J Thorac Cardiovasc Surg 2015;149:1349-55.

10. Yeh T Jr, Ramaciotti C, Leonard SR, Roy L, Nikaidoh H. The aortic translocation (Nikaidoh) procedure: midterm results superior to the Rastelli procedure. J Thorac Cardiovasc Surg 2007;133:461-9. 